Internat. J. Math. \& Math. Sci.

Vol. 24, No. 7 (2000) 461-467

S0161171200002416

(C) Hindawi Publishing Corp.

\title{
UNIFORM CONVERGENCE OF NUMERICAL CONFORMAL MAPPINGS OF INTERIOR DOMAINS IN THE CHARGE SIMULATION METHOD
}

\author{
TETSUO INOUE
}

(Received 20 November 1998)

\begin{abstract}
The uniform convergence of the approximations by new numerical schemes in the charge simulation method, which have been recently proposed by Inoue (1997), will be studied. The exponential decrease of the errors will also be shown.
\end{abstract}

Keywords and phrases. Charge simulation method, numerical conformal mapping, uniform convergence, nonsingularity of matrix.

2000 Mathematics Subject Classification. Primary 41A10, 65 E05.

1. Introduction. The charge simulation method is very useful to obtain the numerical solution of partial differential equations in electrical engineering. The method can be easily applied by solving a system of simultaneous linear equations. Many examples show that the method makes it possible to get rather precise solutions for the boundary value problems with respect to domains bounded by smooth curves $[1,2,3,4,5,6,7]$. However, many parts of the method depend on the results of numerical examples. For instance, the theoretically best distribution of the charge points is not known.

Though a lot of the schemes for computing the approximations of conformal mappings in the charge simulation method have been proposed for interior and exterior domains [1, 2, 3, 4], the uniform convergence of the approximations is not verified even now. For the Dirichlet problem it has been shown by Katsurada-Okamoto [5] and Murota [7]. In fact they have shown the exponential decrease of the errors.

In this paper, the uniform convergence of the approximations by new numerical schemes, which have been recently proposed by Inoue [3, 4], will be studied. The exponential decrease of the errors will also be shown.

2. Numerical schemes. The schemes for the numerical conformal mapping of interior and exterior domains in the charge simulation method have been recently proposed by Amano [1, 2] and Inoue [3, 4]. In this section, the latter is shown, and the uniform convergence of the approximations and the exponential decrease of the errors are studied in the next section.

Let $G$ denote an interior domain whose boundary is a Jordan curve $\gamma$. Without loss of generality, we assume that $G$ contains zero and $\infty$ in its interior and exterior, respectively.

Let $g(z)$ map conformally the unit disk $|w|<1$ onto $G$ with the expansion

$$
g(z)=d z+d_{1} z^{2}+\cdots, \quad d>0
$$


near $z=0$. Then the following scheme for computing an approximation of $g(z)$ has been recently proposed in $[3,4]$.

SCHEME 2.1. The approximation $g_{n}(z)$ of $g(z)$ may be obtained as follows:

(2a) $\left\{z_{n, i}\right\}_{i=1}^{n}$ and $\left\{\zeta_{n, i}\right\}_{i=1}^{n}$ (called charge points and collocation points) are chosen on $|z|=\rho$ and on $|z|=1$, respectively, so that

$$
z_{n, i}=\rho \zeta_{n, i}, \quad \zeta_{n, i}=e^{i \theta_{i}} \quad\left(\rho>1, \theta_{i}=\frac{2 \pi(i-1)}{n}\right) .
$$

(2b) When $\alpha_{i}(i=0,1,2, \ldots, n)$ are the solutions of a system of simultaneous linear equations

$$
\begin{gathered}
\alpha_{0}+\log \left|\zeta_{n, k}\right|+\sum_{i=1}^{n} \alpha_{i} \log \left|1-\frac{\zeta_{n, k}}{z_{n, i}}\right|=\log \left|g\left(\zeta_{n, k}\right)\right| \quad(k=1,2, \ldots, n), \\
\alpha_{1}+\alpha_{2}+\cdots+\alpha_{n}=-1,
\end{gathered}
$$

the charges at $\left\{z_{n, i}\right\}_{i=1}^{n}$ are given by $\left\{\alpha_{i}\right\}_{i=1}^{n}$.

(2c) The approximation $g_{n}(z)$ is represented by

$$
g_{n}(z)=e^{\alpha_{0}} z \exp \left\{\sum_{i=1}^{n} \alpha_{i} \log \left(1-\frac{z}{z_{n, i}}\right)\right\} .
$$

We have, in Scheme 2.1, assumed that the values $\log \left|g\left(\zeta_{n, k}\right)\right|(k=1,2, \ldots, n)$ are known. It is known that the scheme (2.4) has the following mathematical properties.

(2d) The constant terms of schemes have a geometric meaning. The constant term in (2.4) has the approximations of high accuracy

$$
\alpha_{0} \simeq \log d,
$$

when the charge and collocation points are suitably distributed [3, 4].

(2e) The schemes are dual with respect to interior and exterior mappings. This means that there hold (2.4) with (2.3) and

$$
f_{n}(z)=e^{\alpha_{0}} z \exp \left\{\sum_{i=1}^{n} \alpha_{i} \log \left(1-\frac{z_{n, i}}{z}\right)\right\}
$$

with

$$
\alpha_{1}+\alpha_{2}+\cdots+\alpha_{n}=1
$$

for interior and exterior domains, respectively.

(2f) The scheme (2.4) introduced in this paper has the invariant property (whose definition is shown in the next section) under any scaling of domains.

Invariant schemes analogous to ours have recently been proposed for the mapping from a general domain onto a standard one [2]. However they do not have the dual property. 
3. Murota scheme. Let $h(z)$ de a harmonic function on the closed unit disk $|w| \leq$ 1. Murota [6] has recently proposed the following scheme of approximations in the Dirichlet problem.

SCHEME 3.1. The approximation $h_{n}(z)$ of $h(z)$ may be obtained as follows:

(3a) The charge points $\left\{z_{n, i}\right\}_{i=1}^{n}$ and the collocation points $\left\{\zeta_{n, i}\right\}_{i=1}^{n}$ are chosen on $|z|=\rho(\rho>1)$ and on $|z|=1$, respectively, by the method same as (2a).

(3b) When $\alpha_{i}(i=0,1,2, \ldots, n)$ are the solutions of a system of simultaneous linear equations

$$
\begin{gathered}
\beta_{0}+\sum_{i=1}^{n} \beta_{i} \log \left|\zeta_{n, k}-z_{n, i}\right|=h\left(\zeta_{n, k}\right) \quad(k=1,2, \ldots, n), \\
\beta_{1}+\beta_{2}+\cdots+\beta_{n}=0
\end{gathered}
$$

the charges at $\left\{z_{n, i}\right\}_{i=1}^{n}$ are given by $\left\{\beta_{i}\right\}_{i=1}^{n}$.

(3c) The approximation $h_{n}(z)$ is represented by

$$
h_{n}(z)=\beta_{0}+\sum_{i=1}^{n} \beta_{i} \log \left|z-z_{n, i}\right| .
$$

The approximation is superior in the sense that it remains invariant with respect to trivial affine transformations. More precisely, Murota-scheme has the advantage satisfying the following "invariant" property.

$$
z \longrightarrow a z, \quad z_{n, i} \longrightarrow a z_{n, i}, \quad h(z) \longrightarrow h(z)+b
$$

implies

$$
h_{n}(z) \longrightarrow h_{n}(a z), \quad h_{n}(z) \longrightarrow h_{n}(z)+b,
$$

where $a(\neq 0)$ and $b$ are constant. Using the sub-condition (3.2), the invariant property is easily verified.

We may show that the scheme (2.4) introduced in this paper has the invariant property mentioned above without any sub-condition, but

$$
e^{\alpha_{0}} \longrightarrow a e^{\alpha_{0}}
$$

When the charge points and the collocation points are distributed as (2a), (3.1) with (3.2) is transformed to

$$
h_{n}(z)=\beta_{0}+\sum_{i=1}^{n} \beta_{i} \log \left|1-\frac{z}{z_{n, i}}\right|
$$

with

$$
\beta_{1}+\beta_{2}+\cdots+\beta_{n}=0
$$

Under the condition that the function $h(z)$ may have a conformally extension, the following (3d) and (3e) have been verified by Murota [7]. 
(3d) The coefficient matrix in Scheme 3.1 is nonsingular.

(3e) There exist positive constants $c, 0<\tau<1$ and $n_{0}$ such that

$$
\left|h_{n}(z)-h(z)\right| \leq c \tau^{n}, \quad \forall n \geq n_{0},
$$

where $c$ depends on $\rho$.

4. Uniform convergence. Under the condition that the function may have a conformally extension, the following (4a) and (4b) will be shown in this section.

(4a) The coefficient matrix in Scheme 2.1 is nonsingular.

(4b) There exist positive constants $c, 0<\tau<1$ and $n_{0}$ such that

$$
\left|g_{n}(z)-g(z)\right| \leq c \tau^{n}, \quad \forall n \geq n_{0},|z| \leq 1,
$$

where $c$ depends on $\rho$.

(4a) and (4b) for the approximations $g_{n}(z)$ may be shown from (3d) and (3e), and the fact that the uniform convergence of real parts of regular functions implies the one of the imaginal.

Since the coefficient matrices in Schemes 2.1 and 3.1 are same, (4a) is trivial. Next the uniform convergence of the approximations $g_{n}(z)$, obtained by the Scheme 2.1, will be shown as follows.

Equation (2.3) may be transformed to (3.2) and (3.3), respectively, where

$$
\begin{gathered}
h(z)=\log \left|\frac{g(z)}{z}\left(1-\frac{z}{z_{1}}\right)\right|, \\
\beta_{0}=\alpha_{0}, \quad \beta_{1}=\alpha_{1}+1, \quad \beta_{k}=\alpha_{k} \quad(k=1,2, \ldots, n) .
\end{gathered}
$$

The approximation $h_{n}(z)$ has the form (3.7) with (3.8). When

$$
h_{n}(z)=\log \left|\frac{g_{n}(z)}{z}\left(1-\frac{z}{z_{1}}\right)\right|
$$

with (4.3), $g_{n}(z)$ is represented as (2.4) with (2.3).

Now we consider a function

$$
G_{n}(z)=\frac{g_{n}(z)}{g(z)}
$$

analytic on the disk $|z|<\rho$ with the expansion

$$
G_{n}(z)=\frac{e_{0}^{\alpha}}{d}+c_{1} z+\cdots
$$

near $z=0$.

Then using (3.9), (4.2), (4.4), and (4.5) there holds

$$
|\log | G_{n}(z)||=|\log | g_{n}(z)|-\log | g(z)||=|\log | h_{n}(z)|-\log | h(z)|| \leq c \tau^{n} .
$$

Since $G_{n}(0)=e^{\alpha_{0}} / d, \log G_{n}(z)$ is represented as follows (using the representation has been supported by an unknown researcher). 


$$
\begin{aligned}
\log G_{n}(z) & =\log g_{n}(z)-\log g(z) \\
& =\frac{1}{2 \pi} \int_{0}^{2 \pi} \log \left|G_{n}\left(\rho^{\prime} e^{i \theta}\right)\right| \frac{\rho^{\prime} e^{i \theta}+z}{\rho^{\prime} e^{i \theta}-z} d \theta \quad\left(\rho^{\prime}=\frac{\rho+1}{2}\right),
\end{aligned}
$$

which implies for $|z| \leq 1$ that

$$
\left|\log G_{n}(z)\right|=\left|\log g_{n}(z)-\log g(z)\right| \leq \frac{c T^{n}\left(\rho^{\prime}+1\right)}{\rho^{\prime}-1} .
$$

Therefore there exist positive constants $c_{2}, 0<\tau<1$,

$$
\left|g_{n}(z)-g(z)\right| \leq c_{2} \tau^{n}, \quad \forall n \geq n_{0},|z| \leq 1,
$$

where $c_{2}$ depends on $\rho$.

Thus the uniform convergence of the approximations $g_{n}(z)$, obtained by Scheme 2.1, has been verified.

5. Example. The object of this section is to estimate the exponential decrease of the errors in Scheme 2.1 by an example. We consider the function

$$
w=g(z)=\frac{4 z}{4-z^{2}}
$$

which maps conformally the unit disk $|z|<1$ onto a domain $G$ denoted in Figure 5.1.



FIGURE 5.1. The domain $G$.

EXAMPLE 5.1. We distribute the charge points and collocation points as (2.2). We solve a system of simultaneous linear equations (2.3) with $\rho=2,9 \leq n \leq 42$ and obtain the charges $\left\{\alpha_{i}\right\}_{i=1}^{n}$.

Using the charges, the approximation (2.4) is represented. Accuracy of the errors of $g_{n}(z)$ is estimated by the maximum $E(n)$ of

$$
\left|g_{n}\left(\zeta_{100, i}\right)-g\left(\zeta_{100, i}\right)\right| \quad(i=1,2, \ldots, 100) .
$$

By the maximum principle for the regular functions, it is sufficient that the errors are estimated only on the boundary. The graphs $\log E(n)$ are shown in Figures 5.2 and 5.3 for even and odd $n$, respectively. 


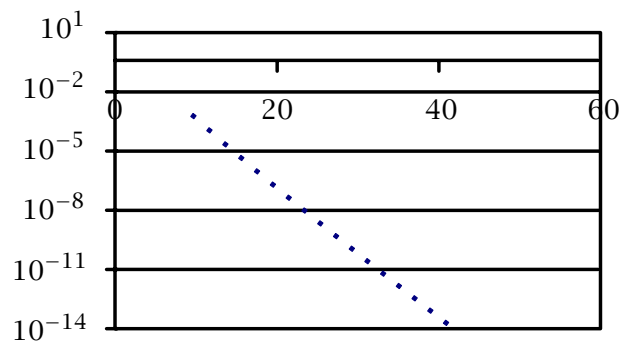

FIGURE 5.2. Graph for even $n$.

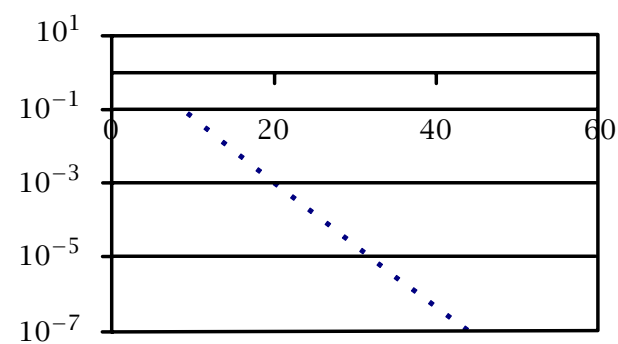

FIGURE 5.3. Graph for odd $n$.

The numerical calculation has been performed in MsDevf90 (PC98XA21-NEC) and with double precision.

6. Concluding remarks. We have assumed that the values $g(z)$ at collocation points are known. Otherwise at first consider the inverse function $z=g^{-1}(w)$ of $w=g(z)$. Then $\left|g^{-1}(w)\right|=1$ holds on the boundary of $G$. Applying the charge simulation method for $z=g^{-1}(w)$, the approximation of $g^{-1}(w)$ will be utilized instead of the real values at the collocation points.

\section{REFERENCES}

[1] K. Amano, A charge simulation method for the numerical conformal mapping of interior, exterior and doubly-connected domains, J. Comput. Appl. Math. 53 (1994), no. 3, 353-370. MR 95i:30007. Zbl 818.30004.

[2] K. Amano and T. Inoue, Dilation invarience of the numerical conformal mapping in the charge simulation method, Trans. Japan Soc. Indust. and Appl. Math. 8 (1998), 1-17 (Japanese).

[3] T. Inoue, Algorithm for computing an inverse function of a conformal mapping by fundamental solution method, Trans. Inform. Process. Soc. Japan 38 (1997), no. 2, 377-379. MR 97m:30005.

[4] T. Inoue and K. Amano, Numerical study based on theoretical distributions of charge points in charge simulation method for numerical conformal mappings, Trans. Japan Soc. Indust. and Appl. Math. 7 (1997), 429-442 (Japanese).

[5] M. Katsurada and H. Okamoto, The collocation points of the fundamental solution method for the potential problem, Comput. Math. Appl. 31 (1996), no. 1, 123-137. CMP 1362 387. Zbl 852.65101. 
[6] S. Murasima, Charge Simulation Method and its Application, Morikita Syuppan, Tokyo, 1993 (Japanese).

[7] K. Murota, On "invariance" of schemes in the fundamental solution method, Trans. Inform. Process. Soc. Japan 3 (1993), 533-535 (Japanese).

Tetsuo Inoue: Department of Applied Mathematics, Kobe Mercantile Marine College, HigASHINADA, FUKAE-MINAMI 5-1-1, KoBE 658-0022, JAPAN

E-mail address: inoue-te@tiedu.kshosen.ac.jp 


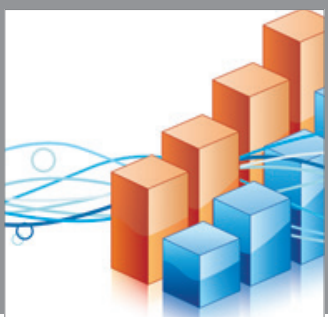

Advances in

Operations Research

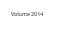

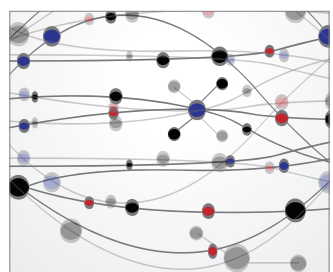

\section{The Scientific} World Journal
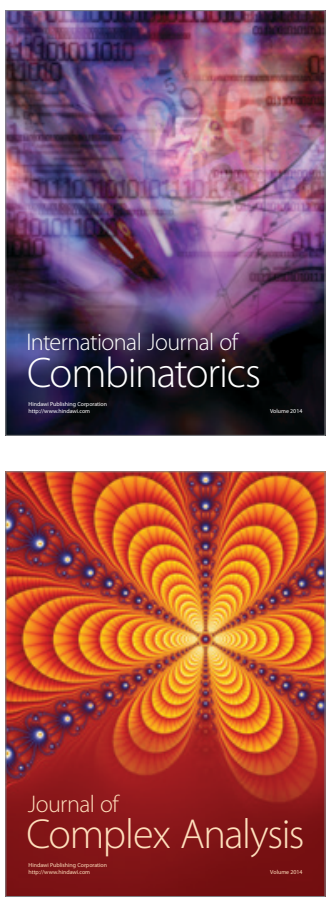

International Journal of

Mathematics and

Mathematical

Sciences


Journal of

Applied Mathematics
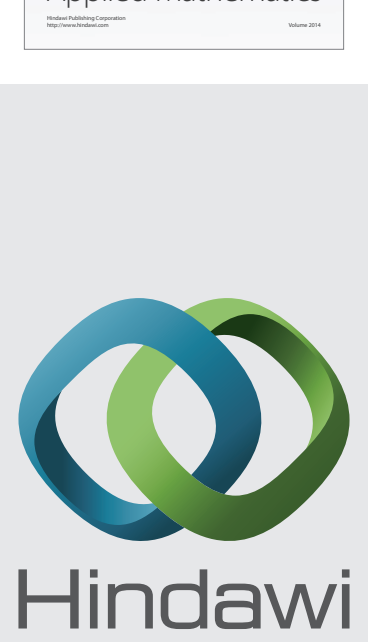

Submit your manuscripts at http://www.hindawi.com


Mathematical Problems in Engineering
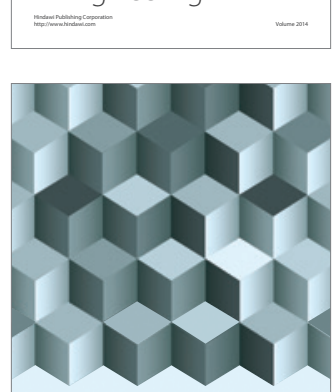

Journal of

Function Spaces
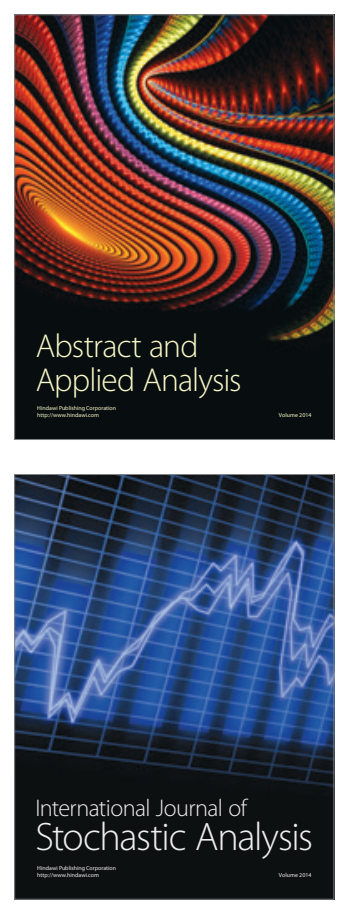

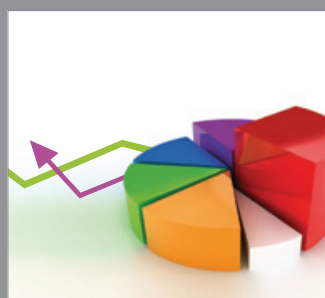

ournal of

Probability and Statistics

Promensencen
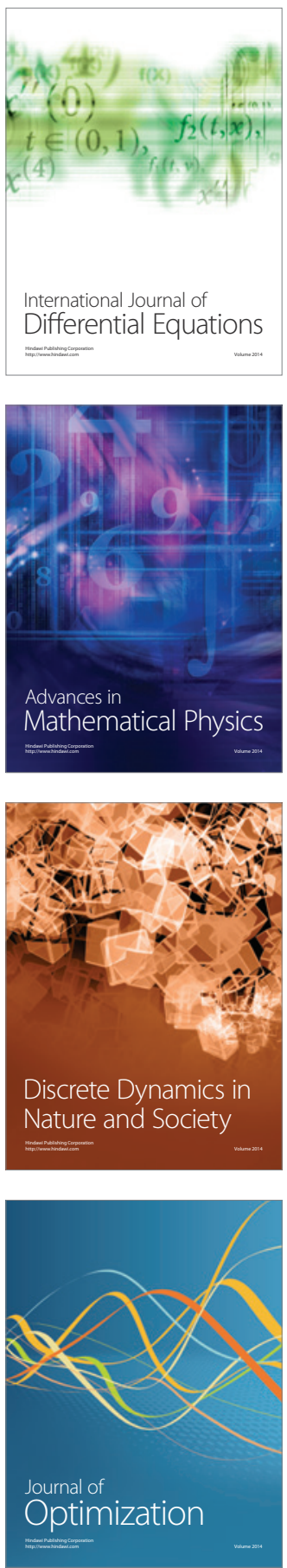